of the foramen ovale.

In most animals of 2-4 days of age, the passage through the foramen ovale can easily be forced, so that double waves actually are observed without forced inflation. However between the age of 4-7 days only the increased pulmonary resistance produced by lungg inflation, provoked opening of the foramen ovale, and above that age no opening was seen. The use of kaolin to absorb the bacteria and to block the small vessels of the brain was suggested by Dr. Pudenz when he visited Japan in 1964 and we appreciated his advice. We have begun experiments in which organisms adsorbed to kaolin or carbon particles are injected in to the femoral vein, while testing the effect of altering pulmonary pressure on the production of brain abscess. A positive result under these conditions would certainly rule out passage of these large particles through the lungs.

Conclusion:

1) Gram negative organisms are commonly responsible for infection in the neonatal period.

2) Prolonged difficult delivery, prematurity and neonatal difficulties are important factors for the development of bacteremia.

3) Opening of the functionally closed foramen ovale produced by pulmonary hypertension, such as results from crying or respiratory difficulties may play an important role in the development of brain abscess or meningitis in the neonatal period.

\title{
17. Intracranial Hematoma in Infants
}

\author{
Takuya TaKagi, Ikuo Mizawa and Minoru Hirayama \\ 2nd Department of Surgery, Nagoya City University Medical School
}

Three cases of post-traumatic intracranial hematoma in infants of age 8,9 and 10 months are reported. The operation revealed that bleeding occured into subdural hydroma in two cases and acute subdural hematoma in one case. Convulsion and vomiting were observed in two cases. Unconsciousness developed in two. Anisocoria was detected only in one but no stiffneck in all cases. Angiograms showed avascular area without shift of the anterior cerebral artery in two cases and fontanelle puncture showed bloody fluid in the other case. There was a linear skull fracture in one case and funduscopic examination revealed subhyaloid hemorrhage in two cases. Surgical intervention resulted in disappearance of symptoms and patients were discharged from the hospital without any abnormal neurological findings. No actual bleeding point was detected at surgery. 